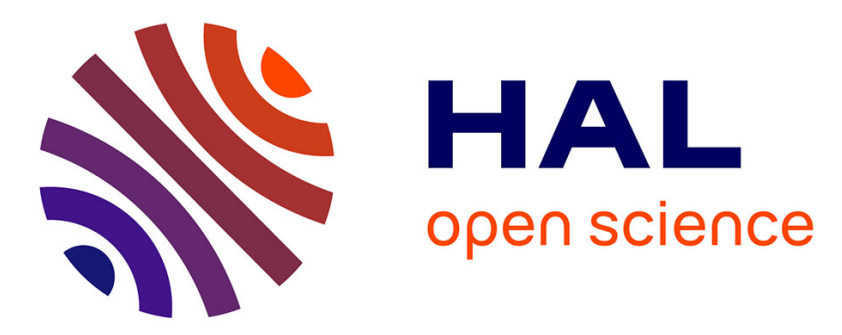

\title{
Computing the expected makespan of task graphs in the presence of silent errors
}

\author{
Henri Casanova, Julien Herrmann, Yves Robert
}

\section{To cite this version:}

Henri Casanova, Julien Herrmann, Yves Robert. Computing the expected makespan of task graphs in the presence of silent errors. Parallel Computing, 2018, 75, pp.41-60. 10.1016/j.parco.2018.03.004 . hal-01968433

\section{HAL Id: hal-01968433 \\ https://hal.inria.fr/hal-01968433}

Submitted on 24 Jan 2019

HAL is a multi-disciplinary open access archive for the deposit and dissemination of scientific research documents, whether they are published or not. The documents may come from teaching and research institutions in France or abroad, or from public or private research centers.
L'archive ouverte pluridisciplinaire HAL, est destinée au dépôt et à la diffusion de documents scientifiques de niveau recherche, publiés ou non, émanant des établissements d'enseignement et de recherche français ou étrangers, des laboratoires publics ou privés. 


\title{
Computing the expected makespan of task graphs in the presence of silent errors
}

\author{
Henri Casanova $^{*, a}$, Julien Herrmann ${ }^{\mathrm{b}}$, Yves Robert ${ }^{\mathrm{c}, \mathrm{d}}$ \\ ${ }^{a}$ University of $\mathrm{Hawai}^{`} i, \mathrm{USA}$ \\ ${ }^{b}$ Georgia Tech, USA \\ ${ }^{c}$ Ecole Normale Superieure de Lyon, France \\ ${ }^{d}$ University of Tennessee Knoxville, USA
}

\begin{abstract}
Applications structured as Directed Acyclic Graphs (DAGs) of tasks occur in many domains, including popular scientific workflows. DAG scheduling has received an enormous amount of attention. Many of the popular DAG scheduling heuristics make scheduling decisions based on path lengths. At large scale compute platforms are subject to various types of failures with no longer negligible probabilities of occurrence. Failures that have recently received increasing attention are "silent errors," which cause data corruption. Tolerating silent errors is done by checking the validity of compute results and re-executing a possibly task from scratch. The execution time of a task then becomes a random variable, and so do path lengths in a DAG. Unfortunately, computing the expected makespan of a DAG (and equivalently computing expected path lengths in a DAG) is a computationally difficult problem. Consequently, designing effective scheduling heuristics in this context is challenging. In this work we propose an algorithm that computes a first order approximation of the expected makespan of a DAG when tasks are subject to silent errors. We find that our proposed approximation outperforms previously proposed approaches both in terms of approximation error and of speed.
\end{abstract}

\section{Introduction}

This paper introduces a new algorithm to approximate the expected makespan of a workflow application, i.e., an application structured as a Directed Acyclic Graph (DAG) of tasks, in which tasks can fail and must be re-executed. A key question when executing workflows on parallel platforms is the scheduling of tasks on the available compute resources, or processors. When not considering task failures, list scheduling algorithms are the de-facto standard [1], and tools are available that use such algorithms for scheduling workflow applications onto large-scale platforms in practice $[2,3]$. The prevalent list scheduling heuristics prioritize tasks with large bottom levels. The bottom-level of a task is defined as

\footnotetext{
A preliminary version of this paper has appeared in the Proceedings of the 2016 IEEE International Workshop on Parallel Programming Models and Systems Software for High-End Computing (P2S2).

${ }^{*}$ Corresponding author
} 
the longest path from that task to the end of the execution, assuming unlimited processors. This heuristic is known as CP-scheduling (Critical Path scheduling $[4,5,6])$, and it has been extended to handle heterogeneous environments (see the HEFT algorithm [7]).

It is well-documented that large-scale platforms are increasingly subject to errors that can cause task failures. In particular, the occurrence of "silent errors" (or SDCs, for Silent Data Corruptions) has been recently identified as one of the major challenges for Exascale [8]. Silent errors can be caused by external causes, including cosmic radiations or packaging pollution. In addition, silent errors can occur when using DVFS (Dynamic Voltage Frequency Scaling) to reduce energy consumption. For instance, at low voltage the probability that a task produces incorrect results increases $[9,10]$.

Regardless of their causes, an effective approach for avoiding propagating results corrupted by silent errors is to use a verification mechanism after executing each task [11]. When an error is detected, the task is then re-executed. The verification mechanism can be general-purpose (e.g., based on replication [12], with re-execution only when the two outputs do not match) or applicationspecific. Many application-specific error detection methods are available for classical High performance Computing (HPC) applications, such as approximate re-execution for ODE and PDE solvers [13], orthogonality checks for Krylovbased sparse solvers $[14,15]$ ), or Algorithm-Based Fault Tolerance (ABFT) for numerical linear algebra [16].

Silent errors make it challenging to define efficient list scheduling algorithms to schedule task graphs. After the first execution of a task, the error detector is used to check the result. If the result is correct, the task's execution is marked as successful, and its successor tasks are marked as ready. If the result is incorrect, then the task must be executed (and, given that the first execution has failed, the second execution will typically succeed with very high probability). While this scheme is conceptually simple, it greatly complicates the computation of the bottom-level of a task. And yet, computing the expected length of the longest path in a DAG with unlimited processors (or equivalently the expected bottomlevel of a task in the DAG), is key to designing silent-error-aware versions of effective list scheduling heuristics (CP-scheduling, HEFT).

It is known that computing the expected length of the longest path in a DAG whose task weights are probabilistic is a difficult problem [6]. Even in the case in which each task is re-executed at most once, i.e., when task weights are random variables taking only two discrete values, the problem remains \#Pcomplete [17] (see Section 2 for a detailed discussion). In this work we develop an algorithm to compute an accurate first-order approximation of the expected length of the longest path in a general DAG in which tasks are subject to silent errors, and whose execution lengths can take two different values, depending upon there is a re-execution or not. More specifically, our contributions are:

- We develop an exact first-order approximation of the expected makespan of a general DAG, which can be computed in polynomial time;

- We compare our approach to two previously proposed approximations for DAGs for three sets of task graphs;

- We quantify approximation errors via comparison to a brute-force Monte Carlo approach; and 
- We show that our proposed approximation (i) leads to lower or similar error than previously proposed approximations (and, importantly, to much lower error when the probability of task failure is low) and (ii) can be computed in practice much faster than previously proposed approximations.

This paper is organized as follows. Section 2 reviews related work. Section 3 formalizes the problem that we address and states assumptions. Section 4 describes our proposed approximation. Section 5 presents evaluation results. Section 6 concludes with a brief summary of results and with perspectives on future work.

\section{Related Work}

In this section we first review previous works on estimating the makespan of probabilistic DAGs, and then review relevant literature on silent errors.

\subsection{Expected makespan of probabilistic 2-state DAGs}

Computing the expected makespan of a DAG whose task execution times obey arbitrary probability distributions is known to be a difficult problem, even with unlimited processors. When task weights are fixed (deterministic), the makespan is the length of the longest path in the graph (also called "critical path"). But assume instead a probabilistic 2-state DAG where task weights obey a simple 2-state probability distribution: task $T_{i}$ has weight $a_{i, 1}$ with probability $p_{i}$ and weight $a_{i, 2}$ with probability $1-p_{i}$. Assume also that all the probability distributions of all tasks are independent. The makespan of the DAG is now a random variable. It is known that computing its probability distribution, or even just its expected value, is a \#P-complete problem [17]. Recall that the class of \#P problems is the class of counting problems corresponding to NP decision problems $[18,19,20]$, and that \#P-complete problems are at least as hard as NP-complete problems.

An informal explanation of why computing the expected makespan of probabilistic 2-state DAGs is a difficult combinatorial problem, is as follows. The main intuitive reason is that the expected value of the maximum of two random variables is not the maximum of their expectations. As a result, when computing the length of a path in the DAG, one must keep track of all possible values for the starting time of each task, together with their probabilities, and there may be an exponential number of such values [21].

In practice, there are three standard methods to compute the expected makespan of a probabilistic 2-state DAG, as described hereafter.

\subsubsection{Monte Carlo simulations}

The Monte Carlo approach works as follows [22, 23]: For each task in the DAG, a value of its weight is sampled from its probability distribution. Once this is done, the DAG is deterministic and its longest path can be computed as explained in Section 3. One then repeats this operation for a large number of trials, generating a new value at each trial. The set of these values empirically approaches the actual distribution of the DAG makespan as the number of trials increases. 
An interesting question is that of determining the number of trials to obtain a high confidence level in the result. We refer the reader to the relevant discussion in [24]. A key drawback of the Monte Carlo approach is that it is computeintensive since the necessary number of trials is typically high. In this work, we only use Monte Carlo to compute a ground truth so as to assess the accuracy of our and previously proposed algorithms that compute approximations of the expected makespan. Hence, instead of determining a minimal number of trials, we conservatively use a very large number of trials so as to guarantee that our ground truth is accurate.

\subsubsection{Approximation by a series-parallel graph}

Basic probability theory tells us how to compute the probability distribution of the sum of two random variables (by a convolution) and of the maximum of two random variables (by taking the product of their cumulative density functions). This simple consideration leads to an exact method to compute the expected makespan when the DAG is series-parallel (see [25] for a definition of series-parallel graphs). The problem with probabilistic 2-state series-parallel graph remains NP-complete in the weak sense and admits a pseudo-polynomial solution [21].

When the DAG is not series-parallel, one approach is to approximate it by a series-parallel graph, which is constructed iteratively, first by a sequence of reductions and then by duplicating some vertices. Dodin's method [26] constructs such an approximated series-parallel graph, whose expected makespan is used to estimate that of the original DAG. See [21, 24] for a detailed description of Dodin's method. We include this method in our quantitative experiments in Section 5.

\subsubsection{Approximation with normality assumption}

The central-limit theorem states that the sum of independent random variables tends to be normally distributed as the number of variables increases. The expected makespan of the DAG is a combination of sums and maximums of the original task weights, so a popular approach proposed by Sculli [27] is based on the normality assumption:

- Approximate the distribution of each task by a normal distribution of same mean and variance. This step has constant cost per task for probabilistic 2-state DAGs.

- Use Clarke's formulas in [28] to compute the mean and variance of the sum and maximum of two (correlated) normal distributions, and then assuming that they also follow normal distributions.

- Traverse the original DAG and compute the mean and variance of the makespan.

See [24] for a full description of Sculli's method, which we we also include in our quantitative experiments in Section 5. 


\subsection{Silent errors}

Considerable efforts have been directed at verification techniques to handle silent errors. A guaranteed, general-purpose verification is only achievable with expensive techniques, such as process replication $[12,29]$ or redundancy [30, 31]. However, application-specific information can be exploited to decrease the verification cost. Algorithm-based fault tolerance (ABFT) $[32,16,33]$ is a wellknown technique to detect errors in linear algebra kernels using checksums. Various techniques have been proposed in other application domains. Benson et al. [13] compare a higher-order scheme with a lower-order one to detect errors in the numerical analysis of ODEs. Sao and Vuduc [15] investigate self-stabilizing corrections after error detection in the conjugate gradient method. Heroux and Hoemmen [34] design a fault-tolerant GMRES capable of converging despite silent errors. Bronevetsky and de Supinski [35] provide a comparative study of detection costs for iterative methods.

Recently, detectors based on data analytics have been proposed to serve as partial verifications [36, 37, 38]. These detectors use interpolation techniques, such as time series prediction and spatial multivariate interpolation, on scientific dataset to offer large error coverage at the expense of a negligible overhead. Although not perfect, the accuracy-to-cost ratios of these techniques tend to be very high, which makes them attractive alternatives at large scale.

The rate of silent errors also depends upon the execution framework. Temperature and power consumption are known to have a high impact [? ]. Furthermore, as mentioned in Section 1, lowering the voltage/frequency is believed to have an adverse effect on system reliability $[9,10]$. In particular, many papers (e.g., $[10,39,40,41])$ have assumed the following exponential error rate model:

$$
\lambda(s)=\lambda_{0} \cdot 10^{\frac{d\left(s_{\max }-s\right)}{s_{\max }-s_{\min }}},
$$

where $\lambda_{0}$ denotes the average error rate at the maximum speed $s_{\max }, d>0$ is a constant indicating the sensitivity of error rate to voltage/frequency scaling, and $s_{\text {min }}$ is the minimum speed. This model suggests that the error rate increases exponentially with decreased processing speed, which is a result of decreasing the voltage/frequency and hence lowering the circuit's critical charge (i.e., the minimum charge required to cause an error in the circuit). This is a concern for resilience, because these studies suggest that minimizing energy via DVFS techniques will also lead to an increased number of silent errors.

A silent error must be detected for each task via some verification mechanism [11]. A naive but general-purpose verification technique would consist in re-running the task once so that an output mismatch indicates a silent error [12]. In this case, the task can be re-executed one extra time so that the correct output can be picked via majority voting. Many application-specific, and thus efficient, error detection methods have been proposed [13, 14, 15, 16]. Because silent errors are not detected when their occur, but only at the end of the execution of the task (by the verification mechanism), the task must be fully re-executed in case an error is detected. Assuming that the task re-execution allows a correct output to be produced, we then need to consider probabilistic 2-state DAGs, in which each task has a weight for the no-error case, and another (longer) weight for the error case. For simplicity, in this work we assume that the weight of a task $T$ is $a$ if there is no silent error, and $2 a$ if a silent error is 
detected (which requires a re-execution). However, our work is immediately extensible to arbitrary such two weights to account, for instance, for the overhead of the silent error detection mechanism.

Finally, this work assume that task failure arrival times are Exponentially distributed with Mean Time Between Failure (MTBF) $1 / \lambda$. Extensions to other probability distributions such as those considered in [? ? ] apply whenever using the distribution MTBF as the (average) inverse of the failure rate and neglecting second order terms (i.e., terms proportional to the square of that failure rate) would lead to accurate approximations of the corresponding failure probabilities.

\section{Problem statement}

We consider a general model of computation in which an application is structured as a Directed Acyclic Graph, in which vertices represent tasks and edges represent task precedence. More formally, let $G=(V, E)$ be a DAG, with $V$ a set of tasks, and $E \subset V \times V$ a set of edges. For each task $i$, let $a_{i}$ be its weight, i.e., its failure-free execution time. Let $p a(i, j)$ denote the length (as a sum of task weights) of the longest path from task $i$ to task $j$, if such a path exists, otherwise let $p a(i, j)=-\infty$. The longest path length in $G$ is then defined as $d(G)=\max _{i, j \in V}\{p a(i, j)\}$. Let us call $d(G)$ the failure-free makespan of $G$. Because $G$ is acyclic, we can compute $d(G)$ in $O(|V|+|E|)$ time as follows: add two zero-weight vertices $v_{1}$ and $v_{2}$ to $G$, where $v_{1}$ represents a unique source task and $v_{2}$ a unique sink task. Also add an edge from $v_{1}$ to any entry task in $G$ (a task without predecessor), and an edge from any exit task in $G$ (a task without successor) to $v_{2}$. Then $d(G)=p a\left(s_{1}, s_{2}\right)$ can be computed in time $O(|V|+|E|)[42$, Section 24.2].

We consider that tasks fail independently and task failure arrival times are exponentially distributed with Mean Time Between Failure (MTBF) $1 / \lambda$. Therefore, the probability that task $i$ fails during its first execution attempt is $1-e^{-\lambda a_{i}}$, in which case the task must be re-executed from scratch.

Our objective is to compute an approximation of the expected makespan of $G$, i.e., the longest path length in $G$ taking into accounts that tasks can fail and must be re-executed. The failure-free makespan defined above is a clear lower bound on the expected makespan.

\section{Approximating the Expected Makespan}

In this section we compute a first-order approximation of the expected makespan of a DAG $G$, which we denote as $\mathcal{E}(G)$. Our approximation relies on the fact that in practice $\lambda$ is close to zero, which allows us to neglect $O\left(\lambda^{2}\right)$ terms.

The probability that the first execution attempt of task $i$ succeeds is:

$$
p_{i}=e^{-\lambda a_{i}}=1-\lambda a_{i}+O\left(\lambda^{2}\right) .
$$

The probability that the first execution attempt of task $i$ fails but that its second execution attempt succeeds is :

$$
\left(1-e^{-\lambda a_{i}}\right) e^{-\lambda a_{i}}=\lambda a_{i}+O\left(\lambda^{2}\right)=1-p_{i}+O\left(\lambda^{2}\right) .
$$


Neglecting the $O\left(\lambda^{2}\right)$ terms leads to the approximation that a task either takes time $a_{i}$, with probability $1-\lambda a_{i}$, or time $2 a_{i}$, with probability $\lambda a_{i}$. In other terms, our first-order approximation consists in assuming that a task never fails more than once. Hereafter when we say that a task fails, we mean that its first execution attempt fails and that its second execution attempt succeeds.

For any $S \subset V$, let $P(S)$ denote the probability that all tasks in $S$ fail and that no task in $V \backslash S$ fails. Let also $L(S)$ denote the length of the longest path in $G$ when all tasks in $S$ fail and no task in $V \backslash S$ fails. $\mathcal{E}(G)$ is thus defined as:

$$
\mathcal{E}(G)=\sum_{S \subset V} P(S) \times L(S) .
$$

We note that:

$$
\begin{aligned}
P(\emptyset) & =\prod_{i \in V}\left(1-\lambda a_{i}+O\left(\lambda^{2}\right)\right)=1-\sum_{i \in V} \lambda a_{i}+O\left(\lambda^{2}\right), \\
P(\{i\}) & =\left(\lambda a_{i}+O\left(\lambda^{2}\right)\right) \times \prod_{j \in V \backslash\{i\}}\left(1-\lambda a_{j}+O\left(\lambda^{2}\right)\right) \\
& =\lambda a_{i}+O\left(\lambda^{2}\right), \text { and } \\
P(S) & =O\left(\lambda^{2}\right) \text { if }|S|>1 .
\end{aligned}
$$

Therefore:

$$
\mathcal{E}(G)=P(\emptyset) \times L(\emptyset)+\sum_{i \in V} P(\{i\}) \times L(\{i\})+O\left(\lambda^{2}\right) .
$$

By definition, $L(\emptyset)=d(G)$ (G's failure-free makespan). Similarly, $L(\{i\})=$ $d\left(G_{i}\right)$, where $G_{i}$ a DAG identical to $G$ but such that task $i$ has weight $2 a_{i}$ instead of weight $a_{i}$. We thus obtain:

$$
\begin{aligned}
\mathcal{E}(G) & =\left(1-\sum_{i \in V} \lambda a_{i}\right) \times d(G)+\sum_{i \in V}\left(\lambda a_{i}\right) * d\left(G_{i}\right)+O\left(\lambda^{2}\right) \\
& =d(G)+\lambda \sum_{i \in V} a_{i}\left(d\left(G_{i}\right)-d(G)\right)+O\left(\lambda^{2}\right) .
\end{aligned}
$$

For a DAG $G=(V, E), d(G)$ can be computed in $O(|V|+|E|)$ time. Therefore, the above approximation can be computed in $O\left(|V|^{2}+|V| \cdot|E|\right)$ time. Lower complexity can be achieved by exploiting the fact that $G$ and the $G_{i}$ 's differ in only the weight of one task.

\section{Evaluation}

\subsection{Makespan Approximation Techniques}

In this section we evaluate three expected makespan approximations techniques:

1. First Order - The approximation described in Section 4;

2. Dodin - The bound in [26], which is computed by transforming any general DAG into an approximately equivalent series-parallel graph and then computing the exact expected makespan of this graph using the approach explained in Section 2.1.2. 


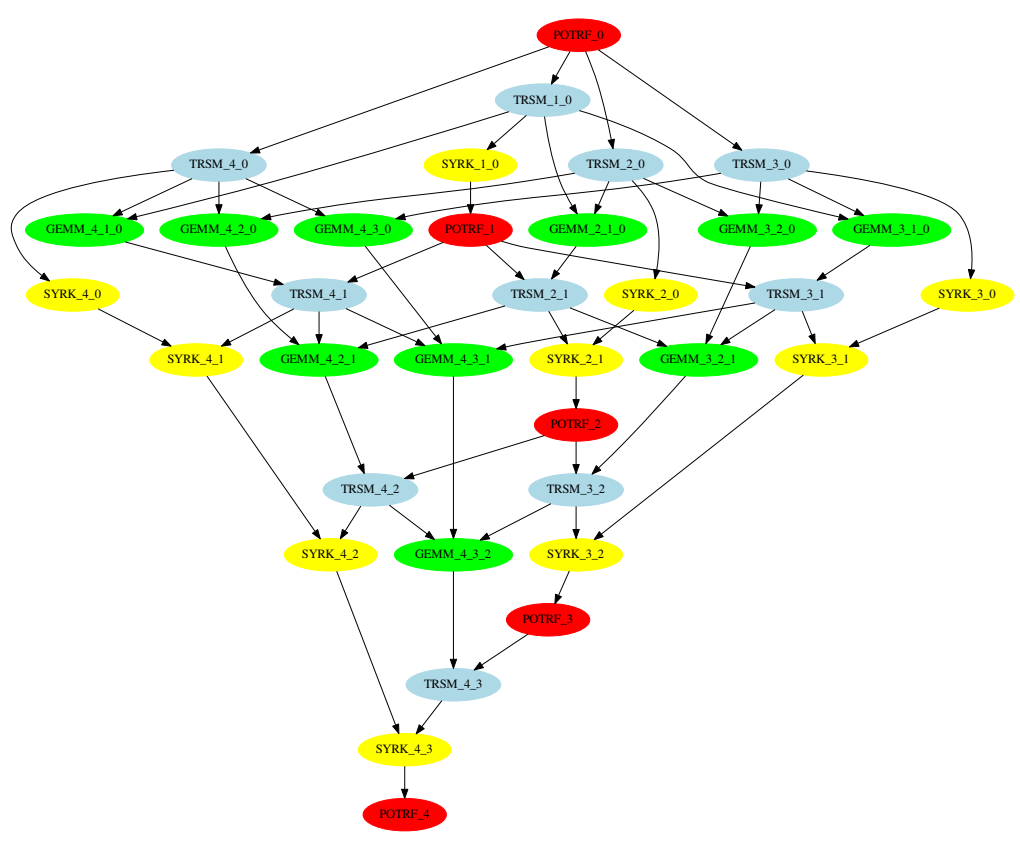

Figure 1: DAG of a Cholesky factorization on a $5 \times 5$ tiled matrix

3. Normal - The approach that consists in approximating the discrete execution time of each task (i.e., $a_{i}$ with probability $p_{i}$ and $2 a_{i}$ with probability $1-p_{i}$ ), by a continuous Normal distribution of same mean and standard deviation. The overall expected makespan in the approximated as explained in Section 2.1.3.

\subsection{Application DAGs}

We measure the error of the three makespan approximation techniques using 3 DAG datasets.

\subsubsection{Linear Algebra}

This dataset consists of DAGs used in numerical linear algebra computations. More specifically, we consider 3 classical factorizations of a $k \times k$ tiled matrix: Cholesky, LU, and QR factorization. Each tile has size $b \times b$, where $b$ is a platform-dependent parameter. Hence the actual size of a $k \times k$ tiled matrix is $N \times N$, where $N=k b$. For each factorization, the number of vertices in the DAG depends on $k$ as follows: the Cholesky DAG has $\frac{1}{3} k^{3}+O\left(k^{2}\right)$ tasks, while the LU and QR DAGs have $\frac{2}{3} k^{3}+O\left(k^{2}\right)$ tasks (but the tasks in QR entail, on average, twice as many floating-point operations as in LU).

Figures 1, 2, and 3 show examples for $k=5$. The tasks in these DAGs are labeled by the corresponding BLAS kernels [43], and their weights are based on actual kernel execution times as reported in [44] for an execution on Nvidia Tesla M2070 GPUs with tiles of size $b=960$. 


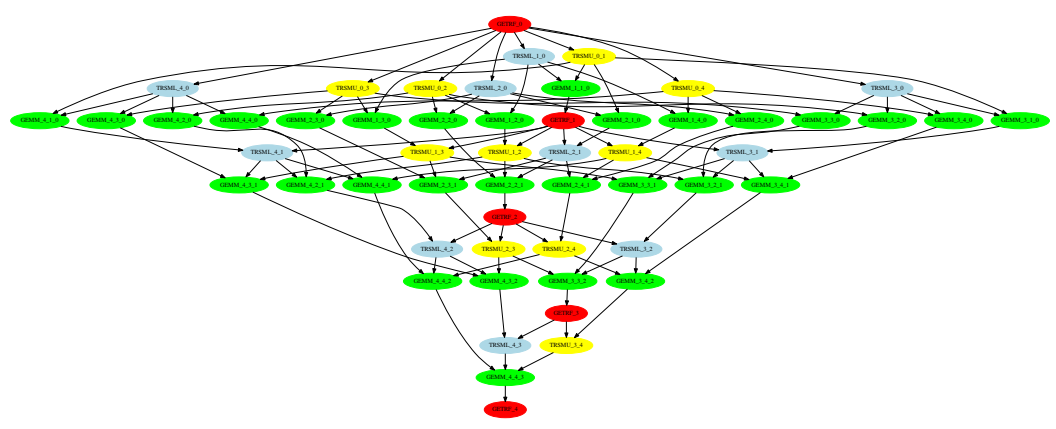

Figure 2: DAG of a LU factorization on a $5 \times 5$ tiled matrix

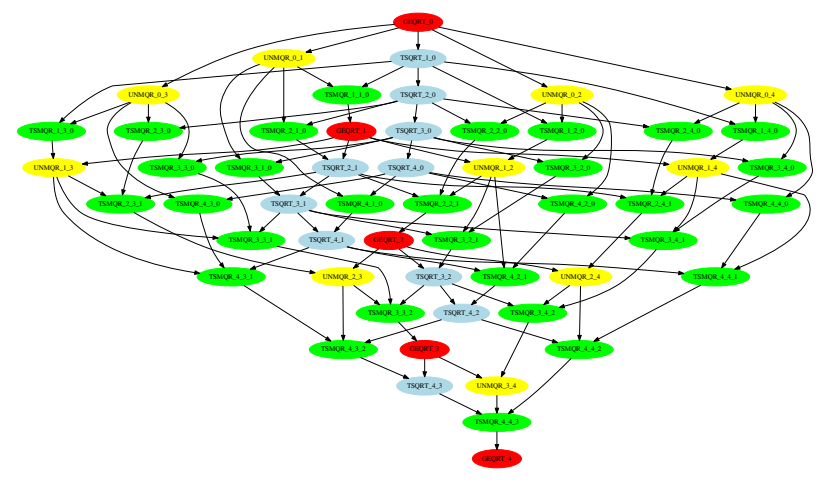

Figure 3: DAG of a QR factorization on a $5 \times 5$ tiled matrix 
For simplicity, in all that follows we call $k$ the DAG size (i.e., the larger $k$ the more tasks). For each DAG class we perform experiments with $k=4,6,8,10,12$, for a total of $3 \times 5=15$ DAGs with up to 650 tasks.

\subsubsection{Assembly Trees}

This dataset consists of assembly trees for a set of sparse matrices obtained from the University of Florida Sparse Matrix Collection (http://www.cise. ufl.edu/research/sparse/matrices/). These matrices satisfy the following assertions: not binary, not corresponding to a graph, square, with a symmetric pattern, with between 20,000 and 2,000,000 rows, with an average number of non-zero elements per row at least equal to 2.5 , and with a total number of non-zero elements at most equal to 5,000,000. Among these matrices, for each group of matrix in the dataset we pick the one that has the largest number of non-zero elements, resulting in 76 matrices (given the content of the dataset at the time this article is being written). We first order the matrices using MeTiS [45] (through the MeshPart toolbox [46]) and amd (available in Matlab), and then build the corresponding elimination trees using the symbfact routine of Matlab. We also perform a relaxed node amalgamation on these elimination trees to create assembly trees. We thus obtain a large set of instances by allowing $1,2,4$, and 16 (if more than $1.6 \times 105$ nodes) relaxed amalgamations per node. Among these assembly trees, we selected four of them for our experiments: 'dump.1.1.amd.Goodwin.rim-447' (a.k.a. 'Goodwin'), 'dump.1.1.amd.Li.li-732' (a.k.a. 'amd.Li.li'), 'dump.1.1.amd.FEMLAB.sme3Dc-932' (a.k.a. 'FEMLAB') and 'dump.1.3.metis.Li.li-732' (a.k.a. 'metis.Li.li').

\subsubsection{Random task graphs}

This dataset consists random DAGs generated using the Directed Acyclic Graph GENerator (DAGGEN) ${ }^{1}$. DAGGEN uses four popular parameters to define the shape of a DAG: size, width, density and jumps.

- The size determines the number of tasks in the DAG (tasks are organized in levels).

- The width determines the maximum parallelism in the DAG, that is the number of tasks in the largest level. A small value leads to "chain" graphs and a large value to "fork-join" graphs.

- The density denotes the number of edges between two levels of the DAG, with a low value leading to few edges and a large value to many edges.

- Random jump edges are added that go from level $l$ to levels $l+1 \ldots l+$ jumps.

The second and third parameters take values between 0 and 1. Our DAG generation procedure is similar to the one used in [47]. Specifically, we generate three sets of random DAGs:

- DAGGen1: 100 randomly generated DAGs with size $=1000$, width $=0.3$, density $=0.5$ and jumps $=5$. A sample DaGgen1 DAG is depicted in Figure 4.

- DAGGEn2: 100 randomly generated DAGs with size $=1000$, width $=0.3$, density $=0.9$ and $j u m p s=7$.

\footnotetext{
${ }^{1}$ Code publicly available at https://github.com/frs69wq/daggen.
} 
- DAGGen3: 100 randomly generated DAGs with size $=1000$, width $=0.9$, density $=0.5$ and jumps $=10$.

\subsection{Experimental Methodology}

For each DAG, and for a given failure rate $\lambda$ (see Section 3), we compute the First Order, Dodin, and Normal approximations of the expected makespan. To compute approximation errors one would ideally use the exact expected makespan (the computation of which is a $\mathrm{P} \#$-complete problem). Instead, we resort to the brute-force Monte Carlo approach described in Section 2.1.1. We use 300,000 random trials. For each task in a trial, the task succeeds or fails as determined by sampling a random time-to-next-failure value from an Exponential distribution with parameter $\lambda$. We then approximate the expected makespan as the average makespan over the 300,000 samples. This method is prohibitively expensive in practice, but provides us with a reasonable ground truth in our experiments. In all results hereafter we report on the relative error between the approximations and this ground truth.

To allow for consistent comparisons of results across different DAGs (with different numbers of tasks and different task weights), in our experiments we simply fix the probability that a task of average weight fails, which we denote as $p_{\text {fail }}$, and compute the failure rate. Formally, for a given DAG $G=(V, E)$ and a given $p_{\text {fail }}$ value, we compute the average task weight as $\bar{a}=\sum_{i \in V} a_{i} /|V|$ and pick the failure rate $\lambda$ such that

$$
p_{\text {fail }}=1-e^{-\lambda \bar{a}} .
$$

We evaluate the performance of our proposed approximation and compare it to its competitors for a range of $p_{\text {fail }}$ values. We present results for a wide range of values, noting that the higher values correspond to error rates much higher than those observed or expected on current and future large-scale computing platforms. The average execution time of a task in our experiments is $\bar{a}=0.15$ seconds. So, for instance, $p_{\text {fail }}=0.01$ leads to an error rate $\lambda=0.067$. The corresponding MTBF is $\mu=1 / \lambda=14.9$ seconds. For a platform with 100,000 processors, this corresponds to an individual MTBF (per processor) of 17.3 days, quite an unrealistic value since individual processor MTBFs are typically estimated to be several years. Table 1 shows the $p_{\text {fail }}$ values used in our experiments and corresponding platform MTBF values (assuming 100,000 processors). The results in the next section show that the lower $p_{\text {fail }}$, the lower the error incurred by our proposed approximation. In particular, our approximation outperforms its competitors for all realistic $p_{\text {fail }}$ values (i.e., $p_{\text {fail }} \leq 0.001$ ), and sometimes even for unrealistically higher values.

\subsection{Approximation Error Results}

In this section we show relative error results, relative to expected makespans computed using the Monte Carlo method. Negative values denote an underestimation, while positive values denote an overestimation. All figures use a logarithmic scale on the vertical axis. 


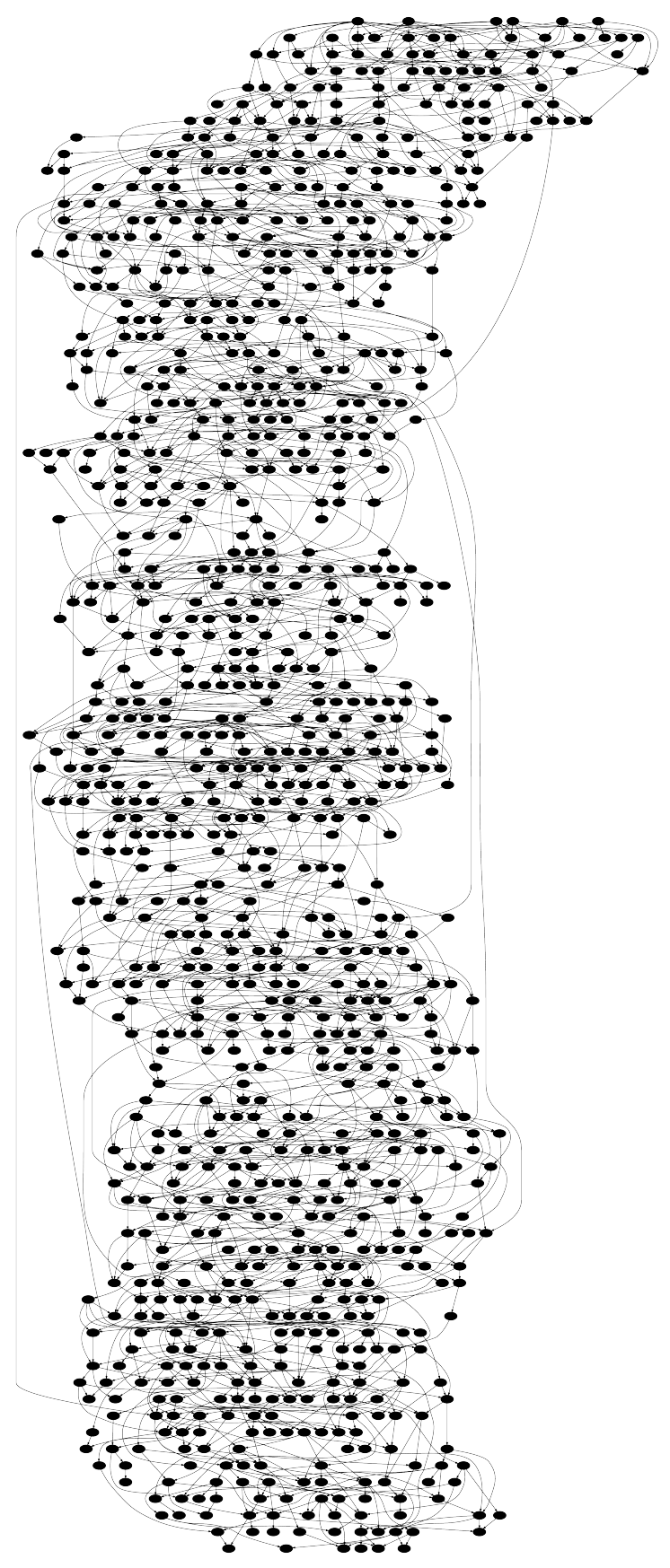

Figure 4: Sample DAGgen1 DAG 
Table 1: $p_{\text {fail }}$ values and corresponding processor MTBF values assuming a 100, 000-processor platform.

\begin{tabular}{|l|l|}
\hline$p_{\text {fail }}$ & processor MTBF \\
\hline 0.01 & 17.3 days \\
0.001 & 173.5 days \\
0.0001 & 4.7 years \\
0.00001 & 47.5 years \\
0.000001 & 475.6 years \\
\hline
\end{tabular}

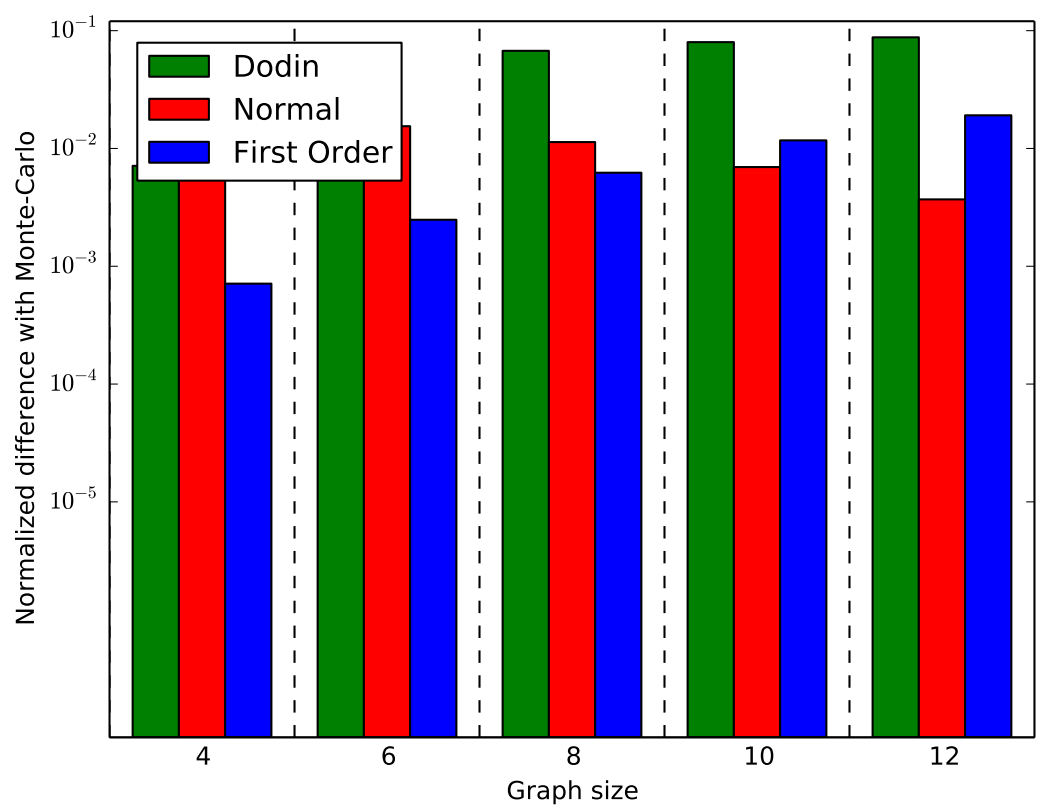

Figure 5: Cholesky $(k=4,6,8,10,12), p_{\text {fail }}=0.01$ 


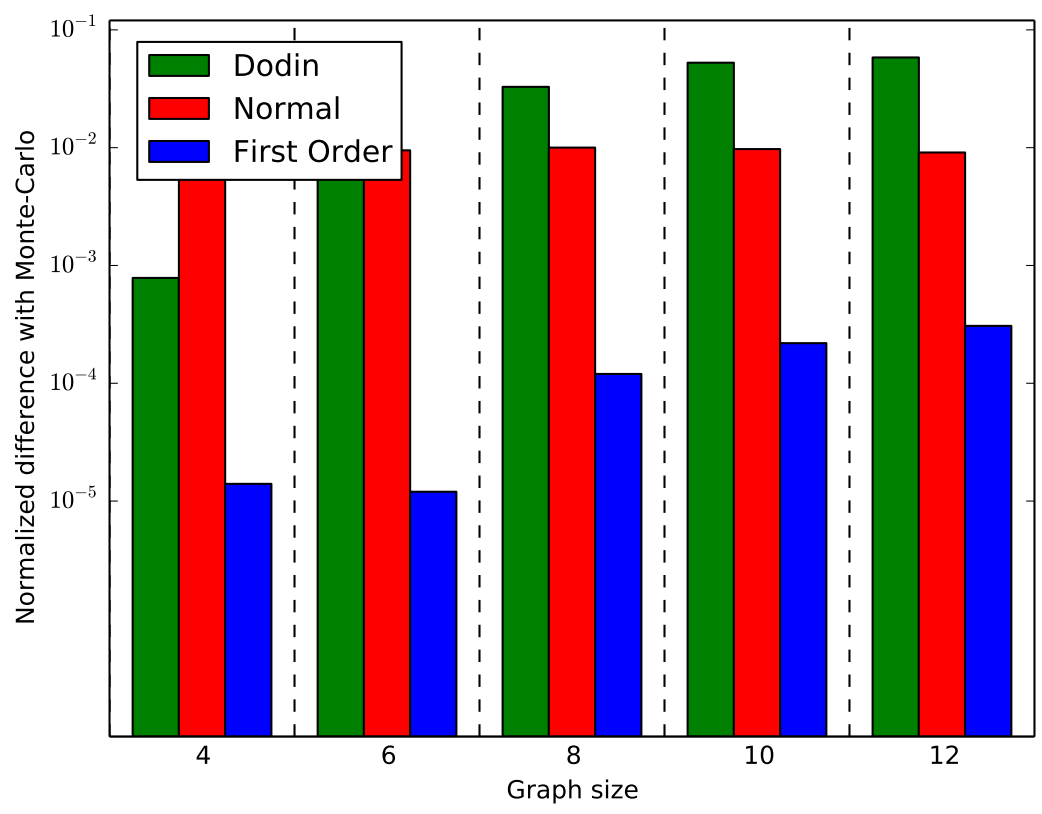

Figure 6: Cholesky $(k=4,6,8,10,12), p_{\text {fail }}=0.001$

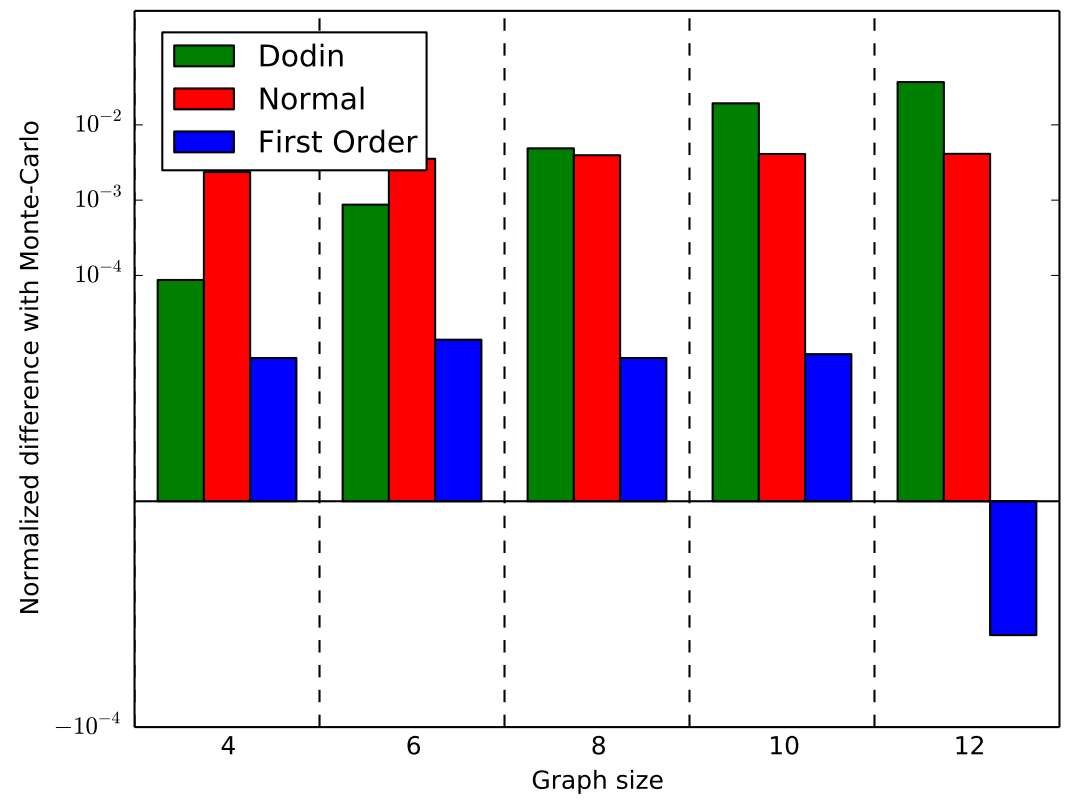

Figure 7: Cholesky $(k=4,6,8,10,12), p_{\text {fail }}=0.0001$ 


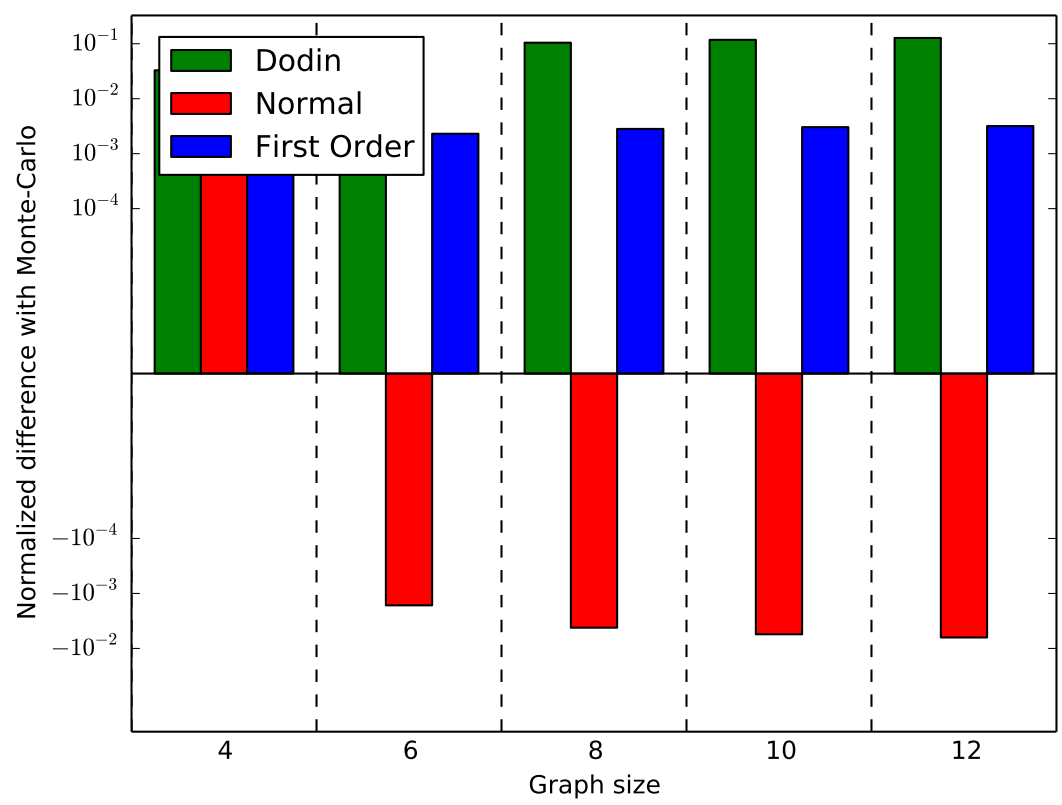

Figure 8: LU $(k=4,6,8,10,12), p_{\text {fail }}=0.01$

\subsubsection{Linear algebra DAGs}

Figures 5, 6, and 7 show relative error vs. graph size for Cholesky graphs and for $p_{\text {fail }}=0.01,0.001$, and 0.0001 , respectively. The data set has 5 graphs representing the Cholesky factorization of a $k \times k$ tiled matrix for $k=4,6,8,10,12$, as described in Section 5.2.1, which correspond to graphs with 20, 56, 120, 220 and 364 nodes. For $p_{\text {fail }}=0.01$, we see that First Order leads to the lowest relative error for graphs sizes below 10, and that Normal leads to the lowest relative error for larger graphs. Dodin leads to the largest relative error, but for the smallest graph size. Considering the largest graph size, First Order has $1.9 \%$ relative error while Normal has $0.3 \%$ relative error and Dodin has $8.7 \%$ relative error. For $p_{\text {fail }}=0.001$, First Order leads to dramatically lower error than its competitors (at least one order of magnitude lower). For instance, for the largest graph, First Order has $0.03 \%$ relative error, compared to $5.8 \%$ for Dodin and $0.9 \%$ for Normal. These results are even more striking for $p_{\text {fail }}=0.0001$. In this case, still for the largest graph, First Order has $-0.0006 \%$ relative error, compared to $3.7 \%$ for Dodin and $0.4 \%$ for Normal. In this case, First Order is an underestimation of the expected makespan.

Figures 8, 9, and 10 show similar results for LU DAGs. The data set has 5 graphs representing the QR factorization of a $k \times k$ tiled matrix for $k=$ $4,6,8,10,12$, as described in Section 5.2.1, which correspond to graphs with $30,91,204,385$ and 650 nodes. The overall message from these results is the same, meaning that First Order leads to drastically lower error than its competitors as $p_{\text {fail }}$ decreases. Here again Dodin leads to the largest errors overall. For $p_{\text {fail }}=0.01$, out approach leads to similar errors of the same order of magnitude as the errors for Normal (and in this case Normal is most often 


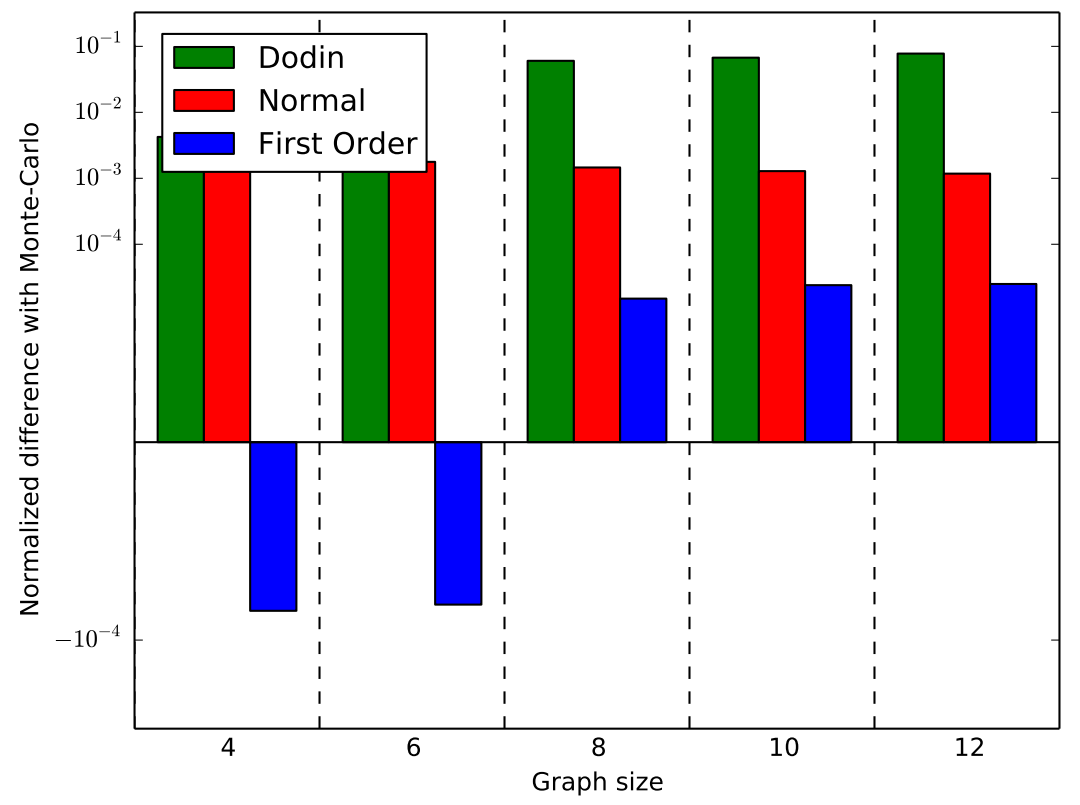

Figure 9: $\mathrm{LU}(k=4,6,8,10,12), p_{\text {fail }}=0.001$

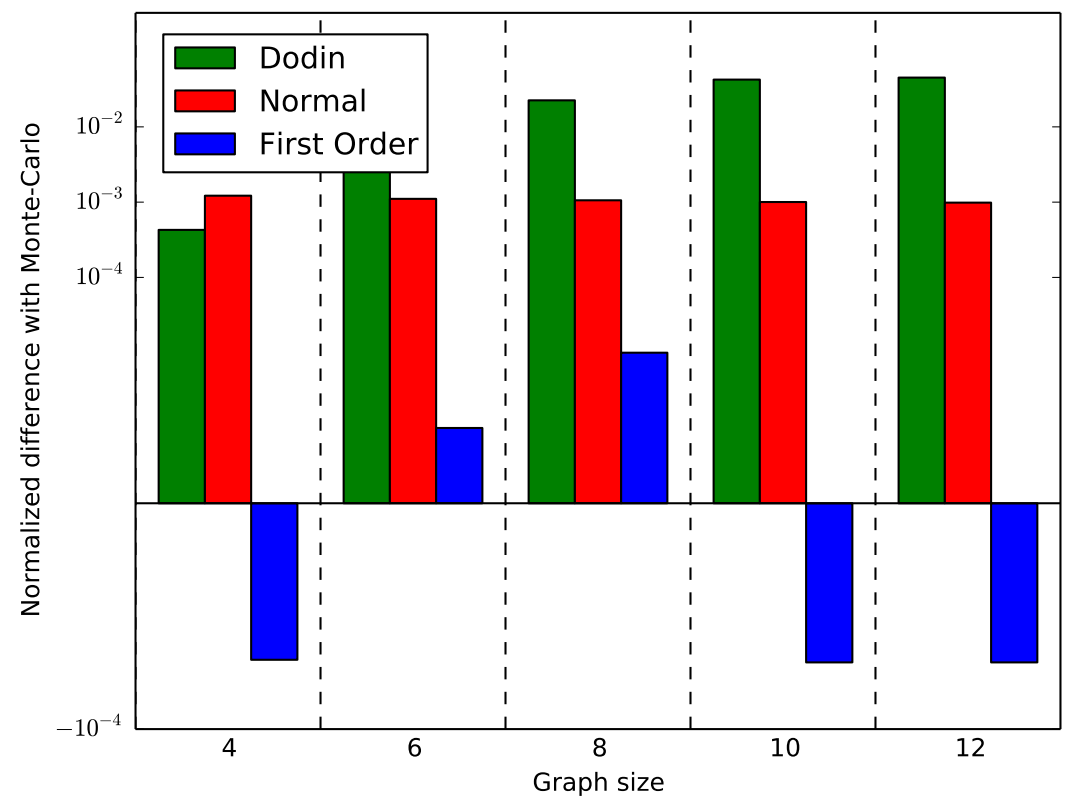

Figure 10: $\mathrm{LU}(k=4,6,8,10,12), p_{\text {fail }}=0.0001$ 


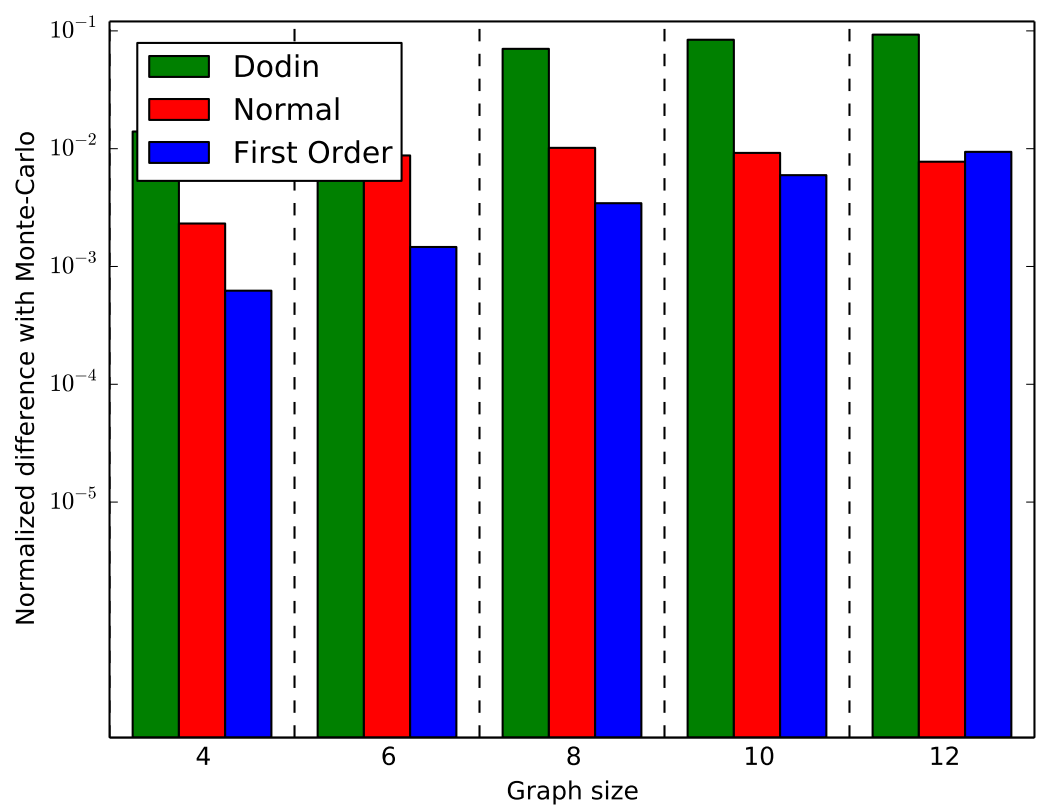

Figure 11: $\mathrm{QR}(k=4,6,8,10,12), p_{\text {fail }}=0.01$

an underestimation while First Order is an overestimation).

Finally, Figures 11, 12, and 13 show results for QR DAGs and similar trends are observed. The data set has 5 graphs representing the QR factorization of a $k \times k$ tiled matrix for $k=4,6,8,10,12$, as described in Section 5.2.1, which correspond to graphs with 30, 91, 204, 385 and 650 nodes. First Order drastically improves on Dodin and Normal for $p_{\text {fail }}=0.001$ and $p_{\text {fail }}=0.0001$. For $p_{\text {fail }}=0.01$, First Order leads to similar or lower error than Normal. Here again, Dodin leads to the highest errors across the board.

\subsubsection{Assembly tree DAGs}

Figures 14 to 18 show results for our Assembly tree DAGs described in Section 5.2.2. These results confirm the general observations from the previous set of results. Here again, Normal always outperforms Dodin. For the low values of $p_{\text {fail }}$ First Order leads to an overestimation of the makespan, while its competitors lead to underestimations, but the magnitude of the estimation errors are comparable. As soon as $p_{\text {fail }} \leq 0.01$, First Order leads to better results that its competitors, and for $p_{\text {fail }} \leq 0.0001$ this improvement is by orders of magnitude (but for the metis.Li.li trees for which its margin of improvement over its competitors is smaller). Overall, we conclude that First Order leads to similar or lower error than Normal (and Dodin), with drastic improvements for lower, more realistic, $p_{\text {fail }}$ values.

\subsubsection{Randomly generated DAGs}

Figure 19 to 23 show results for randomly generated DAGs described in Section 5.2.3. We observe similar trends as for the two previous sets of DAGs. 


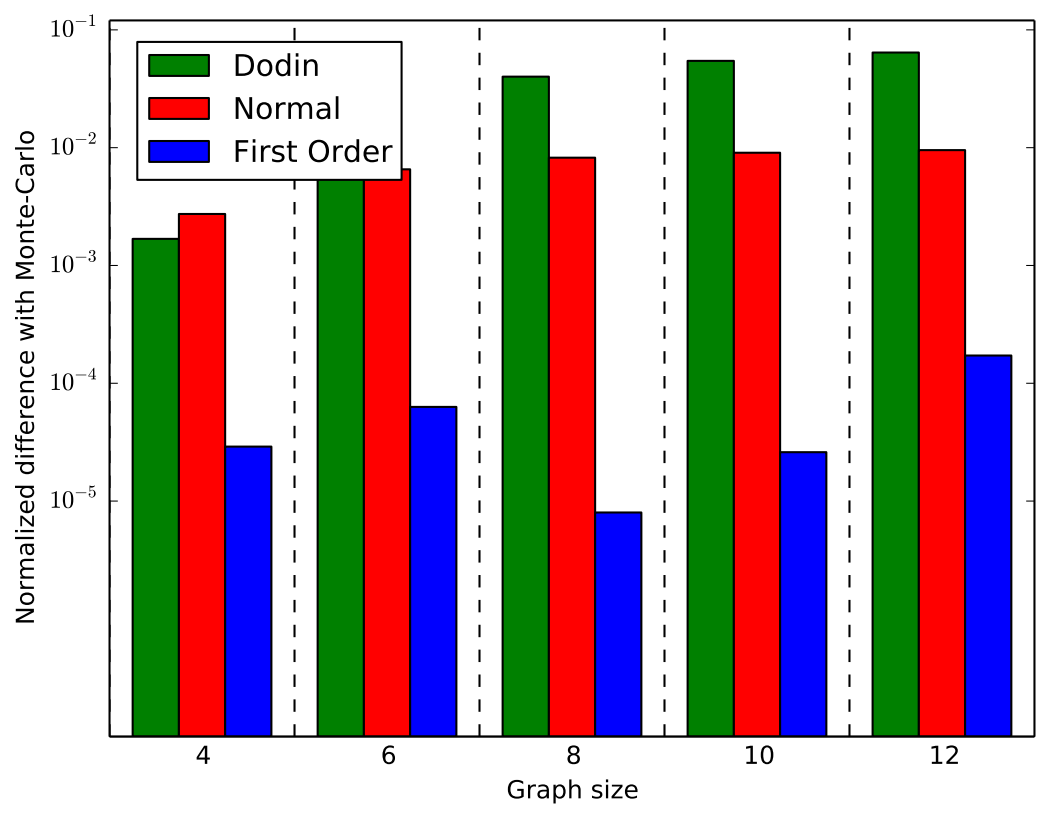

Figure 12: $\mathrm{QR}(k=4,6,8,10,12), p_{\text {fail }}=0.001$

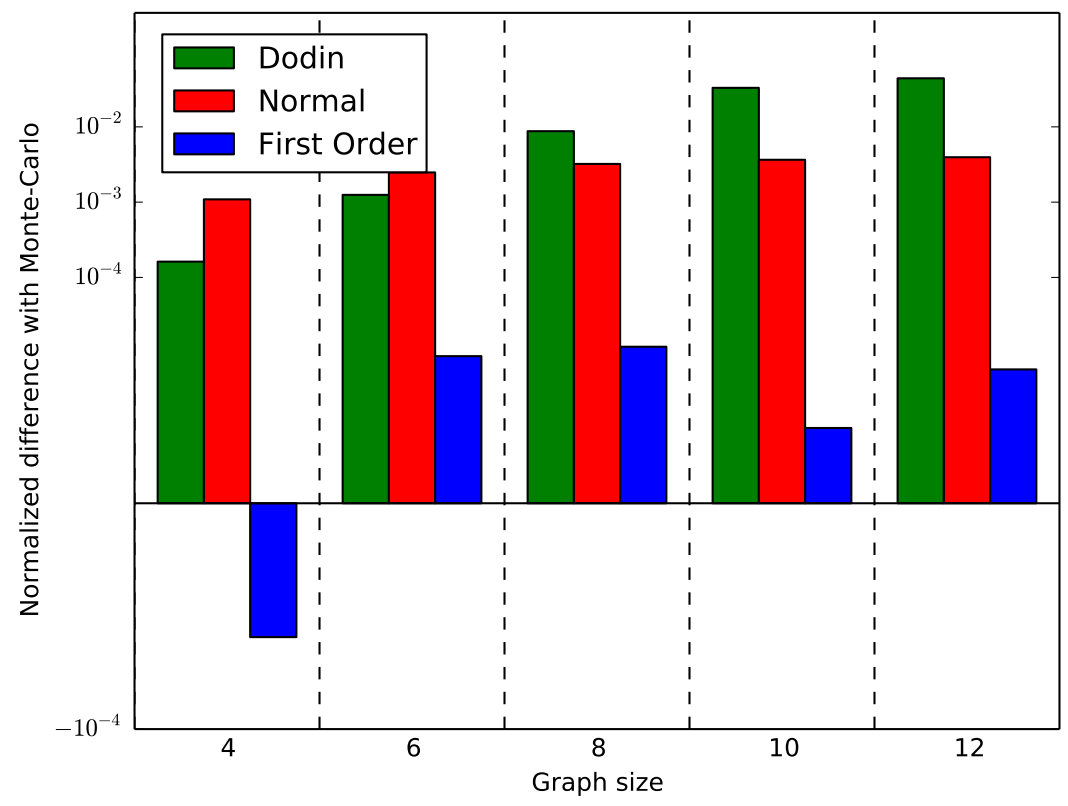

Figure 13: QR $(k=4,6,8,10,12), p_{\text {fail }}=0.0001$ 


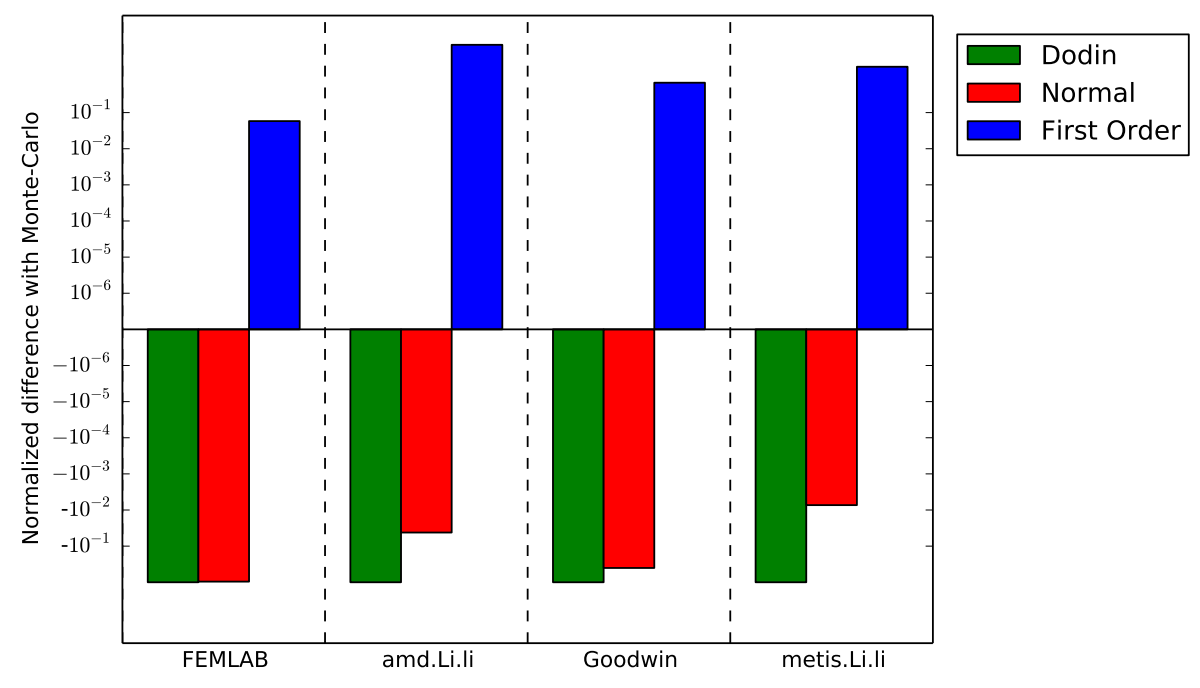

Figure 14: Results on the 4 assembly trees, $p_{\text {fail }}=0.01$

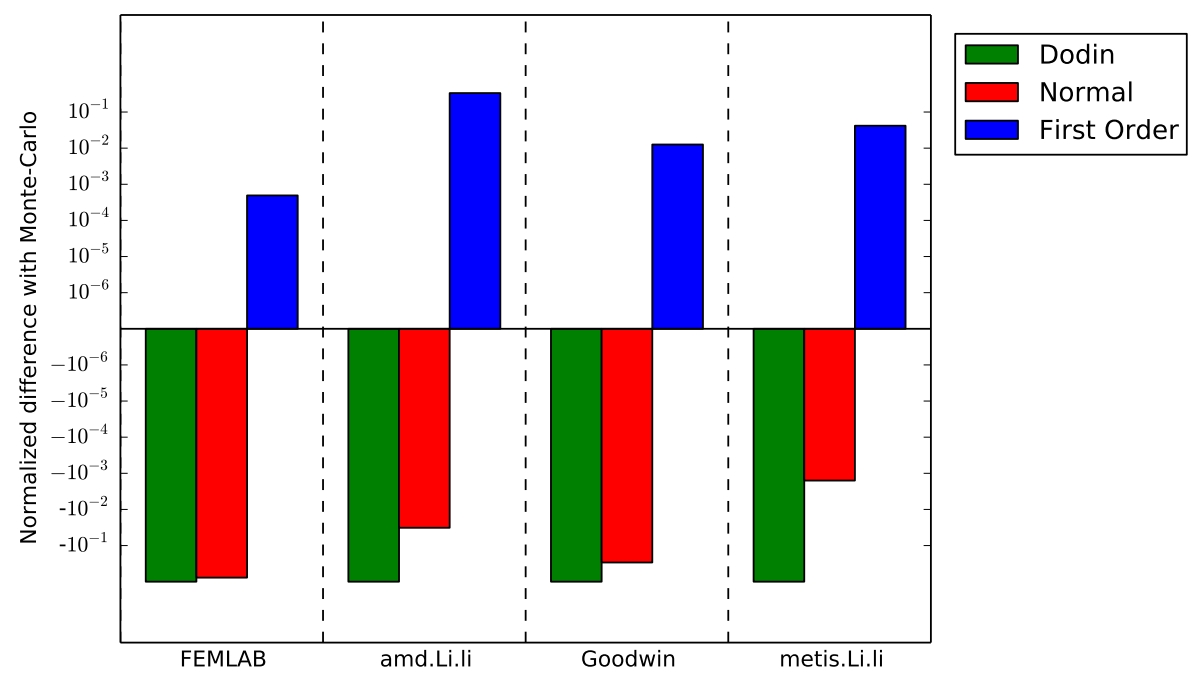

Figure 15: Results on the 4 assembly trees, $p_{\text {fail }}=0.001$ 


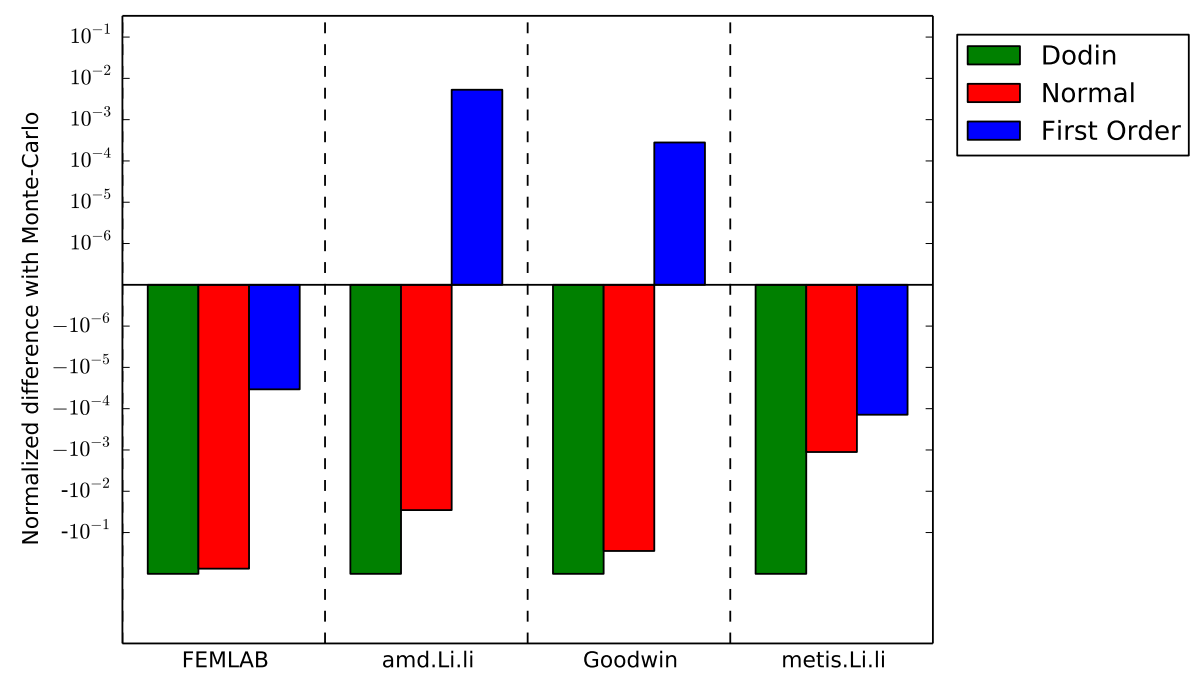

Figure 16: Results on the 4 assembly trees, $p_{\text {fail }}=0.0001$

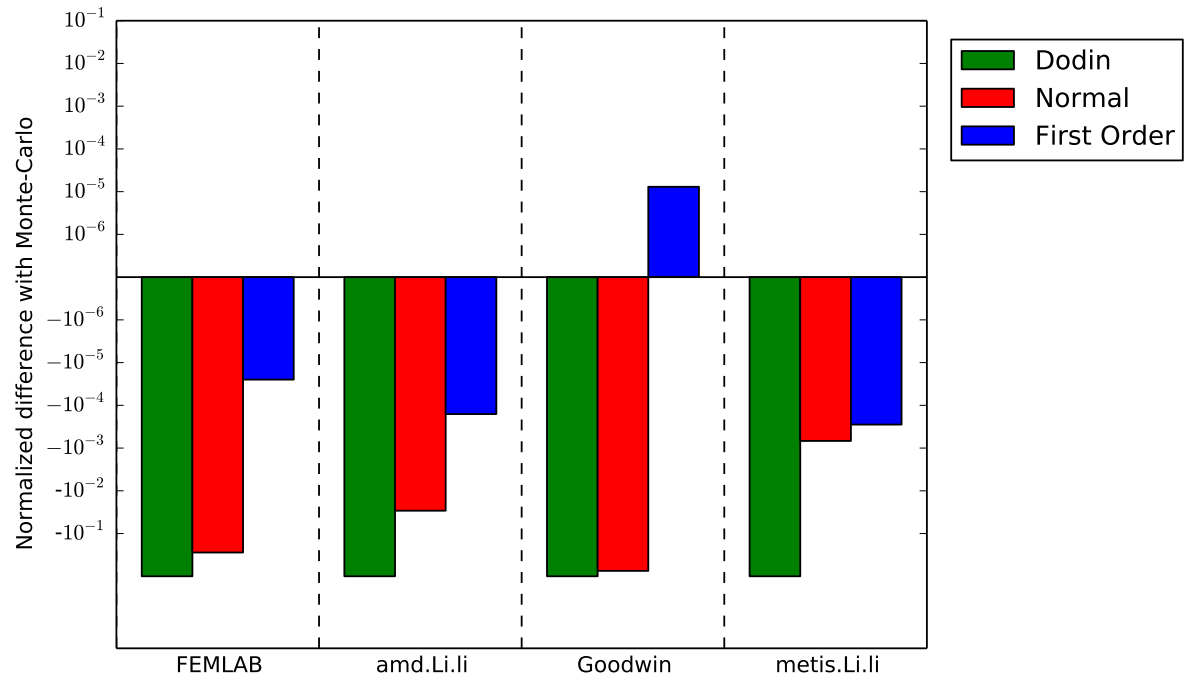

Figure 17: Results on the 4 assembly trees, $p_{\text {fail }}=0.00001$ 


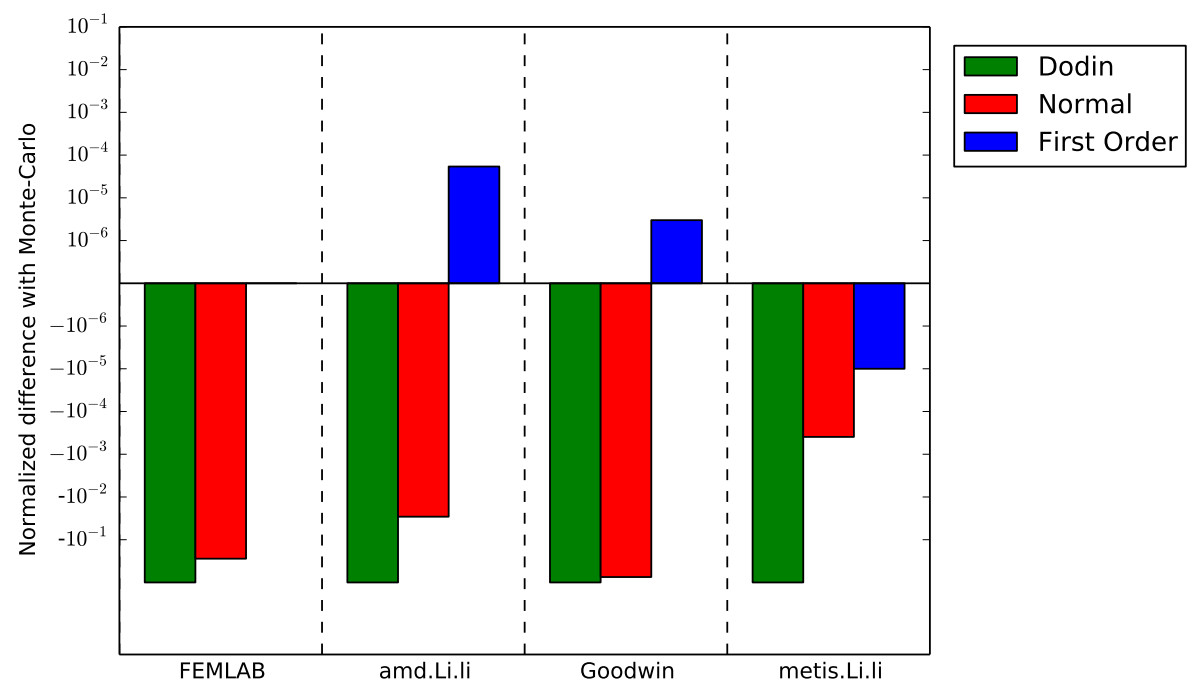

Figure 18: Results on the 4 assembly trees, $p_{\text {fail }}=0.00001$

Normal outperforms Dodin across the board. For low values of $p_{\text {fail }}$, First Order leads to error comparable to that of Normal and Dodin. For $p_{\text {fail }} \leq 0.0001$ error, it leads to orders of magnitude improvements over its competitors. Our overall conclusion is that our proposed approximation approach improves over previously proposed approaches in terms of accuracy, and drastically so for realistically low $p_{\text {fail }}$ values.

\subsection{Scalability Results}

Table 2: Results for a LU DAG with $k=20$ (i.e., 2,870 tasks) and $p_{\text {fail }}=0.0001$.

\begin{tabular}{|l|c|c|c|}
\cline { 2 - 4 } \multicolumn{1}{c|}{} & Dodin & Normal & First Order \\
\hline Error & -0.97 & $954 \times 10^{-6}$ & $7 \times 10^{-6}$ \\
\hline Execution time & $\sim 2$ minutes & $\sim 20$ minutes & $<1$ second \\
\hline
\end{tabular}

To assess the scalability of the three approximation methods, we run experiments with $k=20$ for the LU DAG, i.e., 2, 870 tasks, and $p_{\text {fail }}=0.0001$. Results for other DAGs show similar relative ranking of the three approximation results, and we picked this particular DAG for our scalability analysis because it is from a real-world application, because it is large enough for a scalability study, and because it is small enough that the Monte Carlo method (which provides our ground truth) can run in a reasonable amount of time. For this large graph, we ran the Monte Carlo for ten hours, so as to ensure the accuracy of the ground truth. Error (normalized difference with Monte Carlo) and execution times are shown in Table 2. We see that for this large DAG, Dodin exhibits very large error. First Order is roughly two orders of magnitude more accurate than Normal. We also see that First Order can be computed in under a second, while Normal requires about 20 minutes, i.e., about three orders of magnitude longer. All these experiments are performed on one core of a $2.1 \mathrm{GHz} \mathrm{AMD}$ Opteron(TM) Processor 6272. 


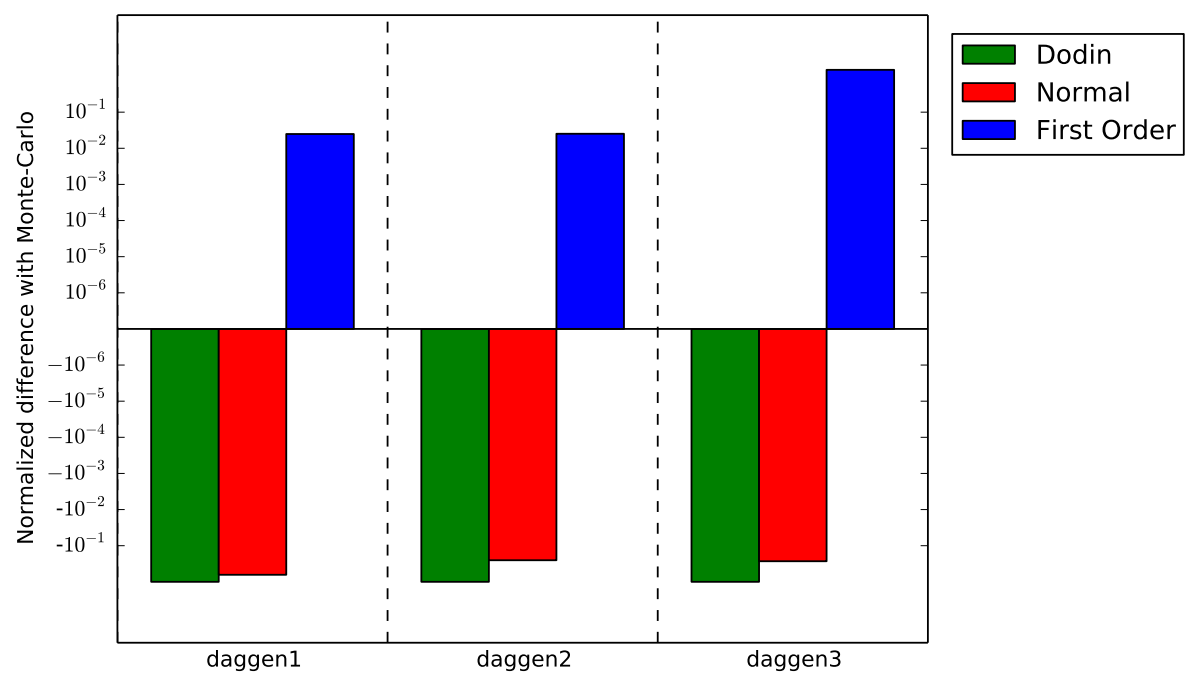

Figure 19: Results on the 3 sets of randomly generated graphs (of size 1000 and various shapes), $p_{\text {fail }}=0.01$

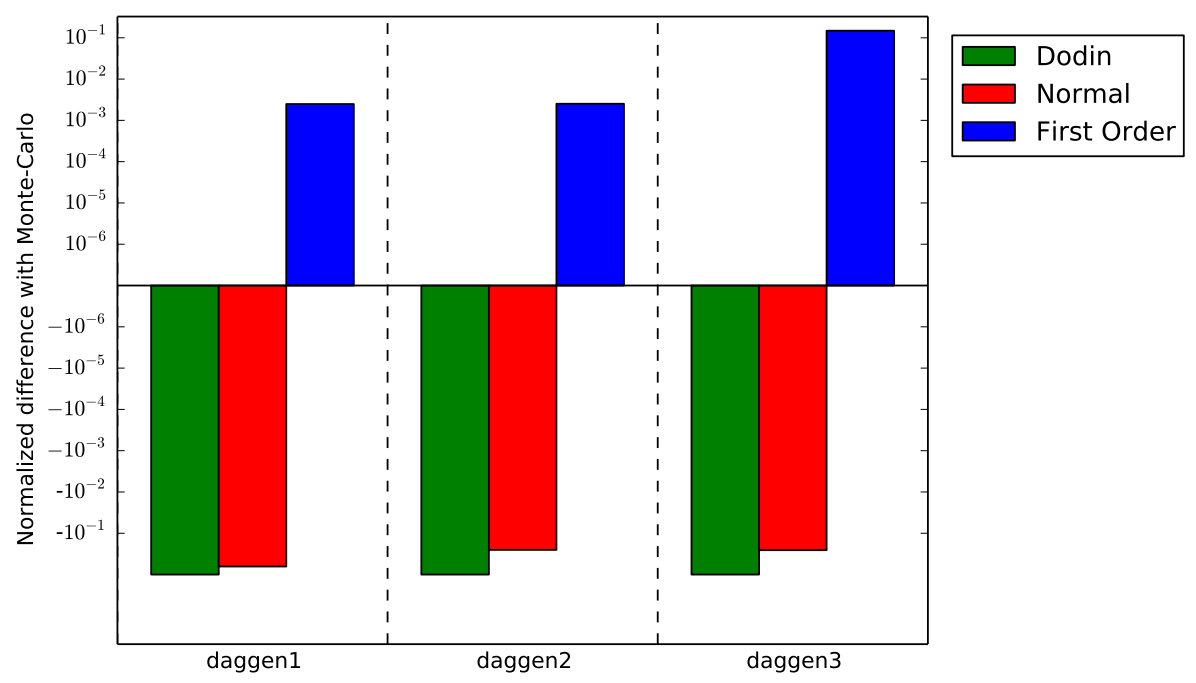

Figure 20: Results on the 3 sets of randomly generated graphs (of size 1000 and various shapes), $p_{\text {fail }}=0.001$ 


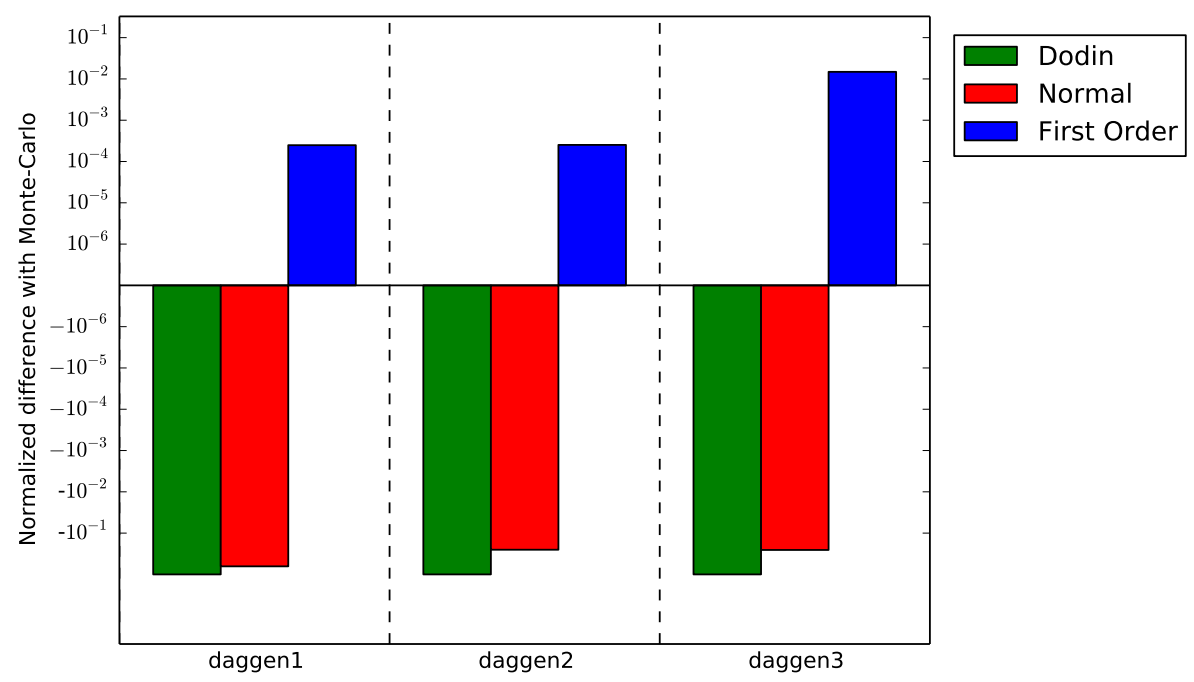

Figure 21: Results on the 3 sets of randomly generated graphs (of size 1000 and various shapes), $p_{\text {fail }}=0.0001$

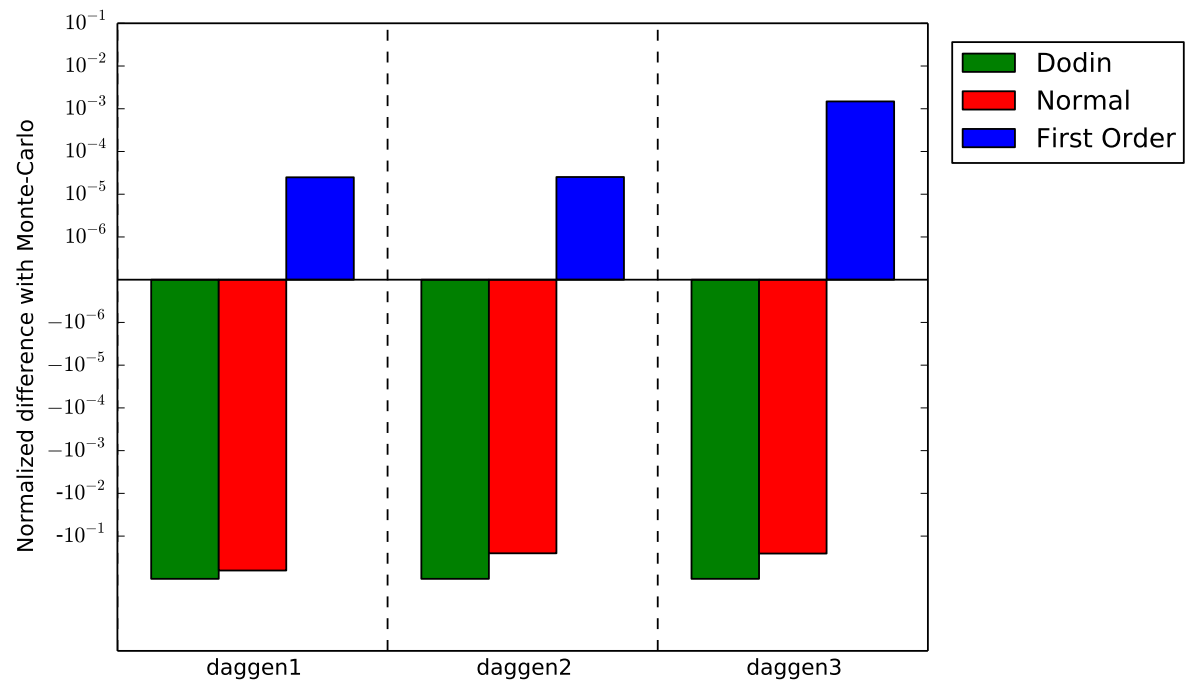

Figure 22: Results on the 3 sets of randomly generated graphs (of size 1000 and various shapes), $p_{\text {fail }}=0.00001$ 


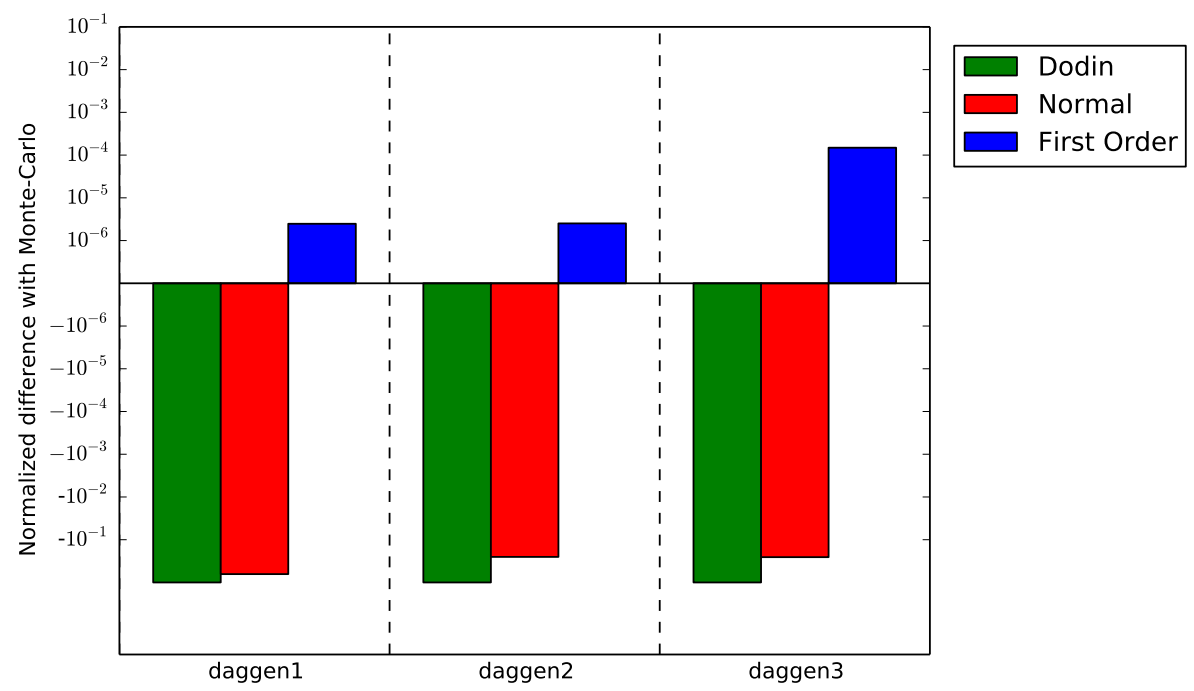

Figure 23: Results on the 3 sets of randomly generated graphs (of size 1000 and various shapes), $p_{\text {fail }}=0.000001$

\subsection{Summary of Results}

Our results show that our proposed approximation, First Order, is accurate as long as the probability of task failure is sufficiently low. In particular, the realistically low probability of task failures it is more accurate that its two competitors by orders of magnitude. When the task failure probability is high, its accuracy is comparable to that of the Normal approximation. Across the board the Dodin approximation leads to the largest error. This is because the DAGs that we consider are far from being series-parallel. As a result, the seriesparallel graph constructed by Dodin is a poor approximation of the original DAG, hence the large errors. Finally, not only is First Order more accurate, but it is also faster. Its computation for a large DAG with 2,870 tasks requires more than three orders of magnitude less time that Dodin and Normal.

\section{Conclusion}

We have proposed an algorithm to compute a first-order approximation of the expected makespan of a Directed Acyclic Graph (DAG) of tasks in which tasks are subject to silent errors that may require task re-execution from scratch due to corrupted results, which we term a failure. Our approximation can be computed in $O\left(V^{2}+V . E\right)$ time for a DAG with $V$ vertices and $E$ edges. It is exact in the first order and neglects second order $O\left(\lambda^{2}\right)$ terms, where $\lambda$ is the exponential failure rate. This amounts to assuming that each task may need to be re-executed at most once. The problem of computing the expected makespan of a DAG of tasks with this assumption is actually a computationally difficult problem (\#P-complete). As a result, techniques to approximate the expected makespan have been proposed in previous works $[26,24]$. We have evaluated our proposed approximation and these previously proposed techniques for three sets of DAGs (linear algebra DAGs, assembly trees, and randomly generated 
DAGs). In our evaluations we quantify the approximation error via comparison to a ground truth computed using a brute-force Monte Carlo method.

Our results show that our proposed approximation is more accurate than previously proposed approximations by several orders of magnitude for realistically low failure rates. In addition, it can be computed much more quickly that these previously proposed approximations, which is crucially important for solving problems at scale. Overall, we have proposed a novel and improved approximation of the expected makespan of probabilistic DAGs in which tasks are subject to silent errors that mandate task re-executions.

A possible future research direction would to use our general approach to compute a more complicated, but still tractable, second order approximation (i.e., an approximation that does not neglect the $O\left(\lambda^{2}\right)$ terms. While the improvement due to including the second order would be likely insignificant for low failure rates, it may be significant for relatively high failure rates, such as those observed for several recent systems [? ? ? ]. These higher failure rates, however, may not be relevant for the scale of current platforms and the failure probabilities of their processors. A broader and more promising future direction is to adapt existing list scheduling scheduling algorithms, or to develop novel such algorithms, that rely on our proposed approximation to make sound scheduling decisions.

Acknowledgments. This work was supported in part by the European project SCoRPiO, by the LABEX MILYON (ANR-10- LABX-0070) of Université de Lyon, within the program "Investissements d'Avenir" (ANR-11-IDEX-0007) operated by the French National Research Agency (ANR), by the PIA ELCI project, and by NSF award SI2-SSE:1642369. The authors would like to thank the reviewers, whose comments and suggestions have helped improve the final version.

\section{References}

[1] Y.-K. Kwok and I. Ahmad, "Benchmarking and comparison of the task graph scheduling algorithms," J. Parallel Distrib. Comput., vol. 59, no. 3, pp. $381-422,1999$.

[2] M. Wieczorek, R. Prodan, and T. Fahringer, "Scheduling of scientific workflows in the askalon grid environment," SIGMOD Rec., vol. 34, no. 3, pp. $56-62,2005$.

[3] E. Deelman, D. Gannon, M. Shields, and I. Taylor, "Workflows and escience: An overview of workflow system features and capabilities," Future Gener. Comput. Syst., vol. 25, no. 5, pp. 528-540, 2009.

[4] P. Brucker, Scheduling Algorithms. Springer-Verlag, 2004.

[5] Y. Robert and F. Vivien, Eds., Introduction to Scheduling. Chapman and Hall/CRC Press, 2009.

[6] M. L. Pinedo, Scheduling: Theory, Algorithms, and Systems, 5th ed. Springer, 2016. 
[7] H. Topcuoglu, S. Hariri, and M. Y. Wu, "Performance-effective and lowcomplexity task scheduling for heterogeneous computing," IEEE Trans. Parallel Distributed Systems, vol. 13, no. 3, pp. 260-274, 2002.

[8] F. Cappello, A. Geist, W. Gropp, S. Kale, B. Kramer, and M. Snir, "Toward exascale resilience: 2014 update," Supercomputing frontiers and innovations, vol. 1, no. 1, 2014.

[9] A. Dixit and A. Wood, "The impact of new technology on soft error rates," in IEEE International on Reliability Physics Symposium (IRPS), 2011, pp. 5B.4.1-5B.4.7.

[10] D. Zhu, R. Melhem, and D. Mosse, "The effects of energy management on reliability in real-time embedded systems," in Proceedings of the IEEE/ACM International Conference on Computer-Aided Design (IC$C A D)$, 2004, pp. 35-40.

[11] T. Hérault and Y. Robert, Eds., Fault-Tolerance Techniques for HighPerformance Computing, ser. Computer Communications and Networks. Springer Verlag, 2015.

[12] D. Fiala, F. Mueller, C. Engelmann, R. Riesen, K. Ferreira, and R. Brightwell, "Detection and correction of silent data corruption for largescale high-performance computing," in Proc. of the ACM/IEEE SC Int. Conf., ser. SC '12. IEEE Computer Society Press, 2012.

[13] A. R. Benson, S. Schmit, and R. Schreiber, "Silent error detection in numerical time-stepping schemes." CoRR, vol. abs/1312.2674, 2013.

[14] Z. Chen, "Online-ABFT: An Online Algorithm Based Fault Tolerance Scheme for Soft Error Detection in Iterative Methods," in Proc. 18th ACM SIGPLAN Symposium on Principles and Practice of Parallel Programming, ser. PPoPP' 13. ACM, 2013, pp. 167-176.

[15] P. Sao and R. Vuduc, "Self-stabilizing iterative solvers," in Proc. ScalA '13. ACM, 2013.

[16] G. Bosilca, R. Delmas, J. Dongarra, and J. Langou, "Algorithm-based fault tolerance applied to high performance computing," J. Parallel and Distributed Computing, vol. 69, no. 4, pp. $410-416,2009$.

[17] J. N. Hagstrom, "Computational complexity of pert problems," Networks, vol. 18 , no. 2 , pp. 139-147, 1988.

[18] L. G. Valiant, "The complexity of enumeration and reliability problems," SIAM J. Comput., vol. 8, no. 3, pp. 410-421, 1979.

[19] J. S. Provan and M. O. Ball, "The complexity of counting cuts and of computing the probability that a graph is connected," SIAM J. Comp., vol. 12 , no. 4 , pp. $777-788,1983$.

[20] H. L. Bodlaender and T. Wolle, "A note on the complexity of network reliability problems," IEEE Trans. Inf. Theory, vol. 47, pp. 1971-1988, 2004. 
[21] R. H. Möhring, "Scheduling under uncertainty: Bounding the makespan distribution," in Computational Discrete Mathematics: Advanced Lectures, H. Alt, Ed. Springer, 2001, pp. 79-97.

[22] M. Mitzenmacher and E. Upfal, Probability and Computing: Randomized Algorithms and Probabilistic Analysis. Cambridge University Press, 2005.

[23] R. M. van Slyke, "Monte carlo methods and the pert problem," Operations Research, vol. 11, no. 5, pp. 839-860, 1963.

[24] L. C. Canon and E. Jeannot, "Correlation-aware heuristics for evaluating the distribution of the longest path length of a DAG with random weights," IEEE Trans. Parallel Distributed Systems, 2016, available at http://doi. ieeecomputersociety.org/10.1109/TPDS.2016.2528983.

[25] J. Valdes, R. E. Tarjan, and E. L. Lawler, "The recognition of series parallel digraphs," in Proc. 11th ACM Symp. on Theory of Computing, ser. STOC '79. ACM, 1979, pp. 1-12.

[26] B. Dodin, "Bounding the project completion time distribution in PERT networks," Operations Research, vol. 33, no. 4, pp. 862-881, 1985.

[27] D. Sculli, "The completion time of PERT networks," The Journal of the Operational Research Society, vol. 34, no. 2, pp. 155-158, 1983.

[28] C. E. Clark, "The greatest of a finite set of random variables," Operations Research, vol. 9, no. 2, pp. 145-162, 1961.

[29] X. Ni, E. Meneses, N. Jain, and L. V. Kalé, "ACR: Automatic Checkpoint/Restart for Soft and Hard Error Protection," in Proc. SC'13. ACM, 2013.

[30] R. E. Lyons and W. Vanderkulk, "The use of triple-modular redundancy to improve computer reliability," IBM J. Res. Dev., vol. 6, no. 2, pp. 200-209, 1962.

[31] J. Elliott, K. Kharbas, D. Fiala, F. Mueller, K. Ferreira, and C. Engelmann, "Combining partial redundancy and checkpointing for HPC," in Proc. ICDCS. IEEE, 2012.

[32] K.-H. Huang and J. A. Abraham, "Algorithm-based fault tolerance for matrix operations," IEEE Trans. Comput., vol. 33, no. 6, pp. 518-528, 1984.

[33] M. Shantharam, S. Srinivasmurthy, and P. Raghavan, "Fault tolerant preconditioned conjugate gradient for sparse linear system solution," in Proc. ICS '12. ACM, 2012.

[34] M. Heroux and M. Hoemmen, "Fault-tolerant iterative methods via selective reliability," Sandia National Laboratories, Research report SAND20113915 C, 2011.

[35] G. Bronevetsky and B. de Supinski, "Soft error vulnerability of iterative linear algebra methods," in Proc. 22nd Int. Conf. on Supercomputing, ser. ICS '08. ACM, 2008, pp. 155-164. 
[36] E. Berrocal, L. Bautista-Gomez, S. Di, Z. Lan, and F. Cappello, "Lightweight silent data corruption detection based on runtime data analysis for HPC applications," in Proc. HPDC, 2015.

[37] L. Bautista Gomez and F. Cappello, "Detecting silent data corruption through data dynamic monitoring for scientific applications," SIGPLAN Notices, vol. 49, no. 8, pp. 381-382, 2014.

[38] — , "Detecting and Correcting Data Corruption in Stencil Applications through Multivariate Interpolation," in Proc.1st Int. Workshop on Fault Tolerant Systems (FTS), 2015.

[39] B. Zhao, H. Aydin, and D. Zhu, "Reliability-aware dynamic voltage scaling for energy-constrained real-time embedded systems," in Proceedings of the IEEE International Conference on Computer Design (ICCD), 2008, pp. 633-639.

[40] G. Aupy, A. Benoit, and Y. Robert, "Energy-aware scheduling under reliability and makespan constraints," in Proceedings of the International Conference on High Performance Computing (HiPC), 2012, pp. 1-10.

[41] A. Das, A. Kumar, B. Veeravalli, C. Bolchini, and A. Miele, "Combined DVFS and mapping exploration for lifetime and soft-error susceptibility improvement in MPSoCs," in Proceedings of the Conference on Design, Automation $\&$ Test in Europe (DATE), 2014, pp. 61:1-61:6.

[42] T. H. Cormen, C. E. Leiserson, R. L. Rivest, and C. Stein, Introduction to Algorithms, 3rd ed. The MIT Press, 2009.

[43] J. Choi, J. Dongarra, S. Ostrouchov, A. Petitet, D. Walker, and R. C. Whaley, "The design and implementation of the ScaLAPACK LU, QR, and Cholesky factorization routines," Scientific Programming, vol. 5, pp. 173-184, 1996.

[44] C. Augonnet, S. Thibault, R. Namyst, and P.-A. Wacrenier, "StarPU: A unified platform for task scheduling on heterogeneous multicore architectures," Concurr. Comput. : Pract. Exper., vol. 23, no. 2, pp. 187-198, 2011.

[45] G. Karypis and V. Kumar, MeTiS: A Software Package for Partitioning Unstructured Graphs, Partitioning Meshes, and Computing Fill-Reducing Orderings of Sparse Matrices Version 4.0, U. of Minnesota, Dpt. of Comp. Sci. and Eng., Army HPC Research Center, Minneapolis, 1998.

[46] J. R. Gilbert, G. L. Miller, and S.-H. Teng, "Geometric Mesh Partitioning: Implementation and Experiments," SIAM Journal on Scientific Computing, vol. 19, no. 6, pp. 2091-2110, 1998.

[47] F. Suter, "Scheduling delta-critical tasks in mixed-parallel applications on a national grid," in Int. Conf. Grid Computing (GRID 2007). IEEE, 2007. 